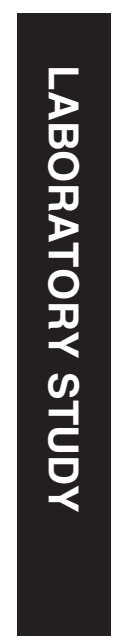

\section{Subretinal fluid is common in experimental non- arteritic anterior ischemic optic neuropathy}

C Yu, JK Ho and YJ Liao
Byers Eye Institute at Stanford, Department of Ophthalmology, Stanford University School of Medicine, Stanford, CA, USA

Correspondence: YJ Liao, Department of Ophthalmology, Stanford University Medical Center, 2452 Watson Court, Palo Alto, CA 94303-5353, USA Tel: +1 6507236995 .

Fax: +1 6507256619

E-mail: yjliao@stanford.edu

Received: 24 March 2014 Accepted in revised form:

7 August 2014

Published online:

26 September 2014
Abstract

Purpose Anterior ischemic optic neuropathy (AION) is an important cause of acute vision loss for which several animal models exist. It has been associated with subretinal fluid in a previous study on patients but not yet so in animal models.

Patients and Methods A patient presented with acute non-arteritic AION (NAION) and underwent ophthalmic evaluation and testing including fluorescein angiography and spectral-domain optical coherence tomography (SD-OCT). On the basis of the patient's findings, we used SD-OCT circular and volume scans to analyze retinal changes in a murine model of NAION.

Results One week after left eye vision loss, the patient had clinical and imaging findings consistent with NAION. On SD-OCT, there was prominent peripapillary retinal thickening consistent with intra-retinal edema and sub-foveolar fluid. Inspired by the findings in human AION, we looked for similar changes in murine NAION using SDOCT. The circular scan did not adequately detect the presence of subretinal fluid. Using the 25-line scan, which covered a larger part of the posterior pole, we found that $100 \%$ of murine AION resulted in subretinal fluid at day 1 . The subretinal fluid resolved by week 1.

Conclusion This study detailed a case of clinical NAION associated with intra-retinal and subretinal fluid. We also found that subretinal fluid was common in murine photochemical thrombosis model of AION and could be found far away from the optic disc. Eye (2014) 28, 1494-1501; doi:10.1038/eye.2014.220; published online 26 September 2014
Introduction

Anterior ischemic optic neuropathy (AION) is the most common acute optic neuropathy in patients older than 50 years and often leads to debilitating and permanent loss of vision. ${ }^{1,2}$ It is generally agreed that non-arteritic AION (NAION) results from acute ischemia of the optic nerve head. Although the pathogenesis is not entirely known, NAION is related to a combination of factors including age, anatomy, vascular supply, compartment syndrome, and hypoxemia during sleep. ${ }^{3}$ There is no current known treatment, but about $40 \%$ of patients can have spontaneous visual improvement in the weeks following onset. $^{4,5}$

Spontaneous visual improvement in NAION may be attributed to different reasons, including resolution of retinal edema and subretinal fluid. A study of 76 patients with AION using OCT demonstrated the presence of subretinal fluid in about half of patients and subfoveal fluid in about $10 \%$ of cases. ${ }^{6}$ A more recent case report has shown similar findings. ${ }^{7}$ Subretinal fluid can collect under the retina in several conditions, generally as a result of more fluid gaining access to the subretinal space than can be removed by physiologic mechanisms. ${ }^{8}$ The clinical significance of the subretinal fluid is unknown. Subretinal fluids are best detected using macular OCT, although macular OCT scans are currently not routinely performed in cases NAION. In recent years, there have been a number of animal models of AION. ${ }^{9}$

Specifically, photochemical thrombosis animal models of focal optic nerve head ischemia seem to reproduce closely the pathology following human NAION. This has been achieved in mice, rats, as well as primates. ${ }^{10-15}$ Subretinal fluid 
has not been described in the animal models of NAION.

Here we report a case of a patient with intra-retinal and subretinal fluid associated with NAION. We then use the murine photochemical thrombosis model of AION to investigate the prevalence of subretinal fluid in murine AION using spectral-domain optical coherence tomography (SD-OCT).

\section{Materials and methods}

We certify that all applicable institutional and governmental regulations concerning the ethical use of human volunteers/animals were followed during this research. All experiments were conducted in accordance with the ARVO Statement for Use of Animals in Ophthalmic and Vision Research and Stanford Administrative Panel on Laboratory Animal Care.

\section{Clinical case}

A 68-year-old man with history of hyperlipidemia presented to the eye clinic after experiencing painless loss of inferior visual field in his left eye for 1 week. He did not have a diagnosis of sleep apnea but has snored constantly for years. We performed a complete history, physical examination, laboratory tests, and ancillary testing including visual field, SD-OCT (Cirrus HD-OCT, Carl Zeiss Meditec, Jena, Germany), fluorescein angiography, and fundus photography.

\section{Animals}

Wild-type C57BL/6 mice were purchased (Charles River, Wilmington, MA, USA) and housed in 12-h light 12-h dark cycle with food and water ad libitum. For in vivo experiments, mice were anesthetized with an intraperitoneal injection of a combination of ketamine (20 mg/kg) (Phoenix Scientific, Saint Joseph, MO, USA) and xylazine $(6 \mathrm{mg} / \mathrm{kg}$ ) (Phoenix Scientific). The pupils of anesthetized mice were dilated with $1 \%$ tropicamide (Alcon Laboratories Inc., Fort Worth, TX, USA) and 2.5\% phenylephrine hydrochloride (Akorn Inc., Lake Forest, IL, USA).

\section{Murine photochemical thrombosis AION model}

We used a mouse photochemical thrombosis model of NAION that we have previously described, ${ }^{14,15}$ and we have performed this injury model in hundreds of adult mice. To induce optic nerve head ischemia, we injected rose bengal (1.25 $\mathrm{mM}$ in phosphate-buffered saline, $2 \mathrm{ml} / \mathrm{kg}$ animal weight) intravenously through the tail vein, then exposed the optic disc to 15 consecutive laser spots with $400-\mu \mathrm{m}$ diameter, $50-\mathrm{mW}$ power, and 1-s duration using a frequency-doubled $532 \mathrm{~nm} \mathrm{Nd:YAG}$ laser (Pascal, OptiMedica, Sunnyvale, CA, USA). For each animal, we induced AION in one eye, and the contralateral eye served as control.

\section{In vivo SD-OCT and analysis of subretinal fluid}

On day 1, week 1, 2, and 3 after AION induction, the mice were anesthetized and imaged using SD-OCT (Spectralis, Heidelberg, Germany). The digital highmagnification lens (Volk Optical Inc., Mentor, OH, USA) was mounted in the front to improve clarity of images. Each eye was lubricated with artificial tears and covered with a custom-made contact lens made with a custom punch and Aclar film (Ted Pella, Inc., Redding, CA, USA). ${ }^{16}$ The SD-OCT circular scan mode consisted of a diameter of $12^{\circ}$ centered at the optic nerve head. The volume scan consisted of 25 horizontal B-scans spanning a $15^{\circ} \times 15^{\circ}$ area centered on the disc. The presence of subretinal fluid was qualitatively graded as small, moderate and large amounts. The location of the subretinal fluid was noted to be inferior, superior, or both in reference to the center of the optic disc. We calculated the volume of subretinal fluid by analyzing the area of fluid in each horizontal B-scan multiplied by the distance between consecutive scans using Adobe Creative Suite (San Jose, CA, USA).

\section{Statistical analysis}

Statistical significance was determined using a paired Student's $t$-test and the Wilcoxon signed-rank test (Prism 6, GraphPad Software, Inc., La Jolla, CA, USA). The level of significance was set at $P<0.05$.

\section{Results}

\section{Clinical case of non-arteritic AION}

A 68-year-old man with history of hyperlipidemia presented with painless inferior visual field loss in the left eye. His best corrected visual acuity was 20/40 in both eyes, and he had a left relative afferent pupillary defect. His anterior and posterior segment exam was significant for $360^{\circ}$ of optic disc swelling in the left eye, most significant superiorly and least significant temporally (Figures 1a and b). There were two small peripapillary flame hemorrhages nasally and marked narrowing of retinal arteries, especially superiorly. The contralateral, right optic disc was significant for C/D ratio of 0 .

Static perimetry using a Humphrey's automated perimeter (30-2 threshold, SITA-Standard) revealed an inferior altitudinal visual defect and superior peripheral 

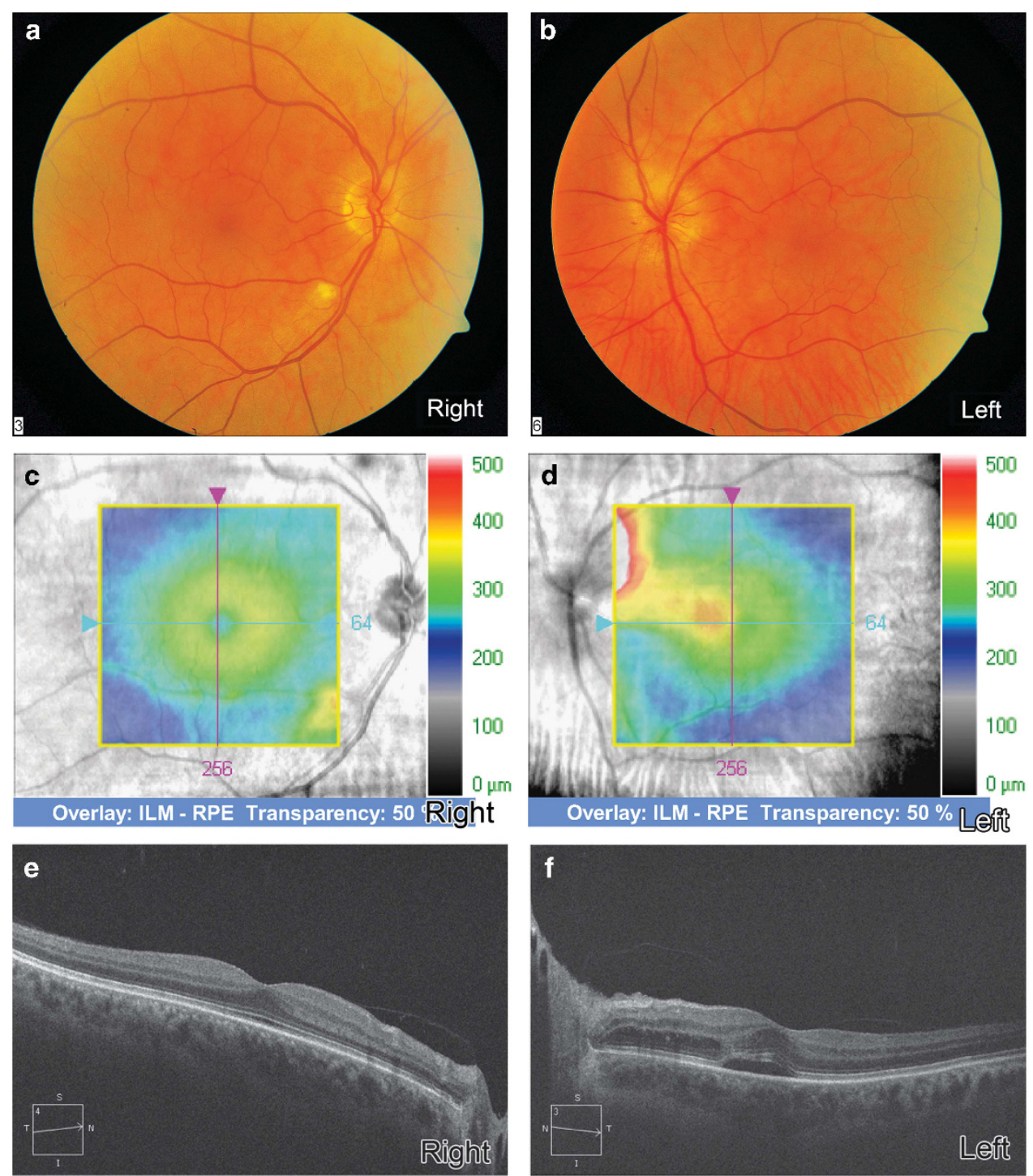

Figure 1 Clinical case of peripapillary retinal edema and subretinal fluid associated with non-arteritic anterior ischemic optic neuropathy. A 68-year-old man woke up with severe vision loss in his left eye. These scans were obtained at 1 week after onset of symptoms. Fundus photography showing diffusely blurred optic disc margin in the left eye (b), contrasting with the normal right eye (a). (c-f) SD-OCT macular volume scans revealed thickening of the total retinal thickness of the peripapillary retina and the nasal macula in the left eye (d) compared with the right eye (c). Individual B-scan through the fovea demonstrated peripapillary intra-retinal edema, which greatly distorted the macular contour, and sub-foveolar fluid in the left eye (f). The right eye (e) had normal macular contour.

constriction in the left eye and normal visual field in the right eye (Figure 2a). On SD-OCT circular scan, all layers of the peripapillary retina, including the retinal nerve fiber layer, appeared diffusely thickened in the left eye. On line scan through the optic disc and fovea, there was evidence of intra-retinal edema, best seen as hyporeflectance of the outer retina, distortion of the normal macular contour, and an increase in the total retinal thickness in the macula just temporal to the disc (Figure 1f). The macular volume scan also showed that there was sub-foveolar fluid. His fluorescein angiogram revealed early staining of the left optic disc and patchy choroidal hypoperfusion extending off the optic disc (Figure $2 b$ ). In the late phase, there was intense left optic disc hyperfluorescence (Figure 2c).

\section{Murine experimental AION}

After induction of AION in mice, there were similar findings as in human AION. Fluorescein angiography performed $30 \mathrm{~min}$ following acute $\mathrm{AION}$ revealed intense staining of the optic disc in the AION eye (Figure 3). On day 1, the circular SD-OCT scans of the peripapillary retina showed no evidence of subretinal 
a

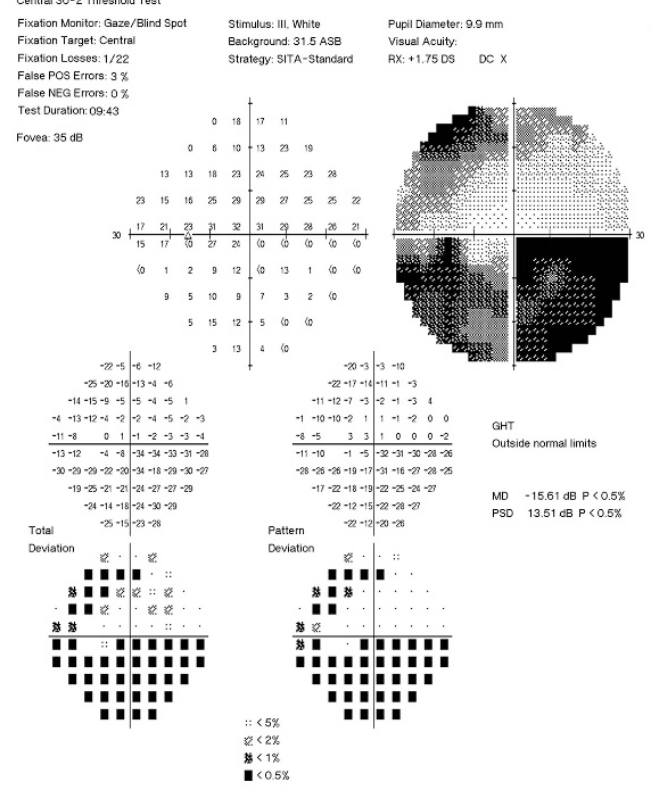

b

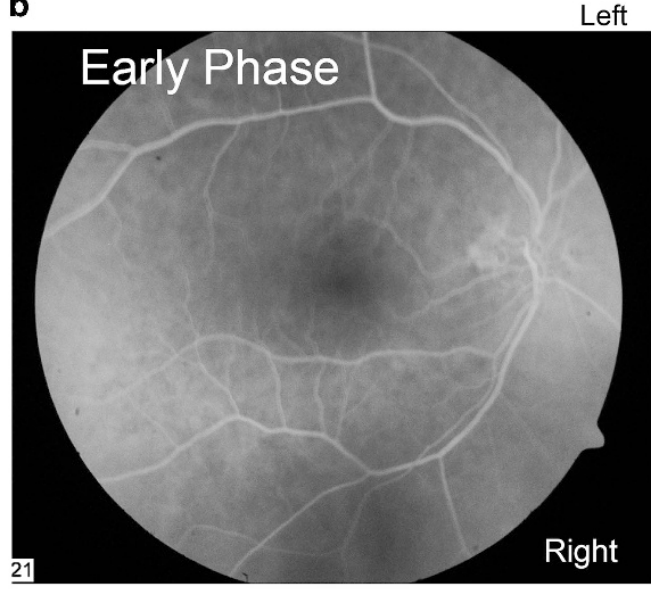

c

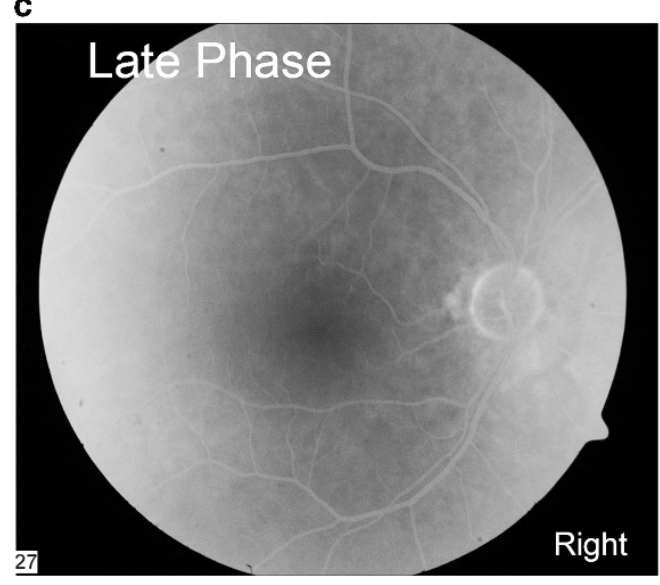

Left Fixation Monitor: Gaze/ Birid Spot
Fixasion Target Central Fixation Troget Central
Fixalon Loseses $0 / 17$ False POS Errors: $4 \%$ False NEG Errors: $0 \%$ Test Duration:

Fovea 36 dB

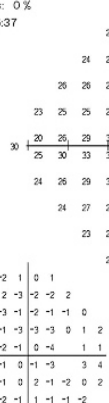

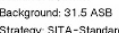

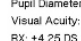

(n)

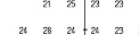

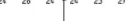

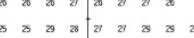

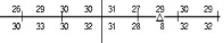

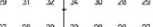

"

$x x=x$

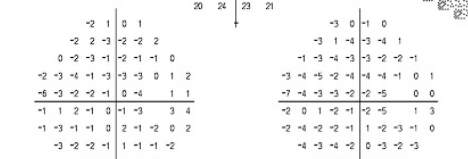

Total
Deviation

Pattern
Deviation

GHT

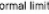

$\begin{array}{ll}\text { MO } & -1200 \mathrm{~dB} \\ \text { PSD } & 222 \mathrm{~dB}\end{array}$

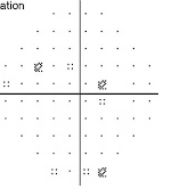

$3-3 \cdot-1 \cdot 2 \cdot-3$

no

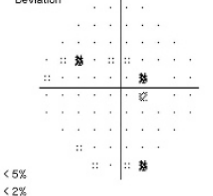

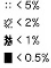

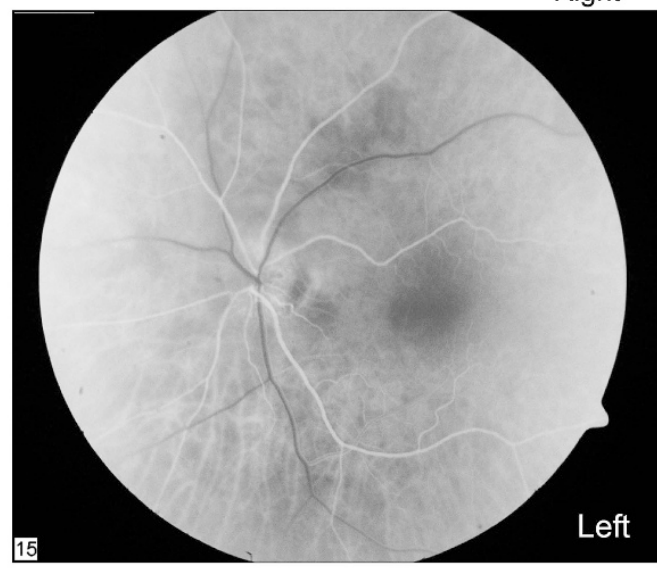

Right

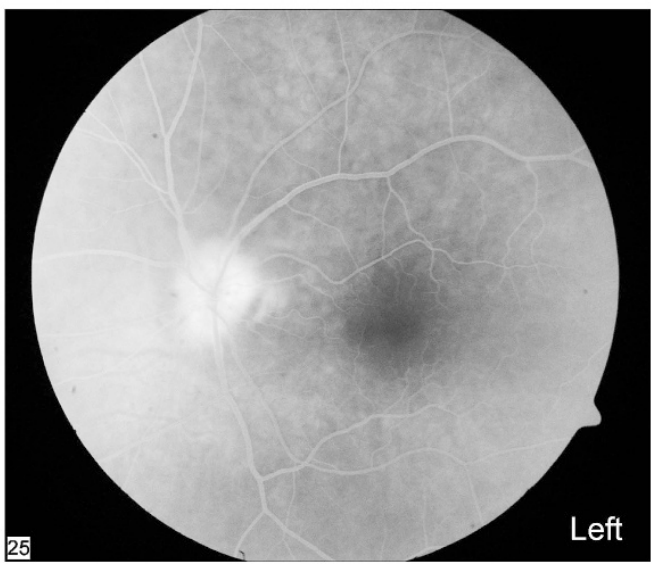

Figure 2 Ancillary testing of the same patient with AION. (a) Humphrey visual field 30-2 test revealed inferior altitudinal visual field defect and superior peripheral constriction in the left eye. (b, c) Fluorescein angiography demonstrated early (b) and late (c) staining of the optic disc in the left eye. 

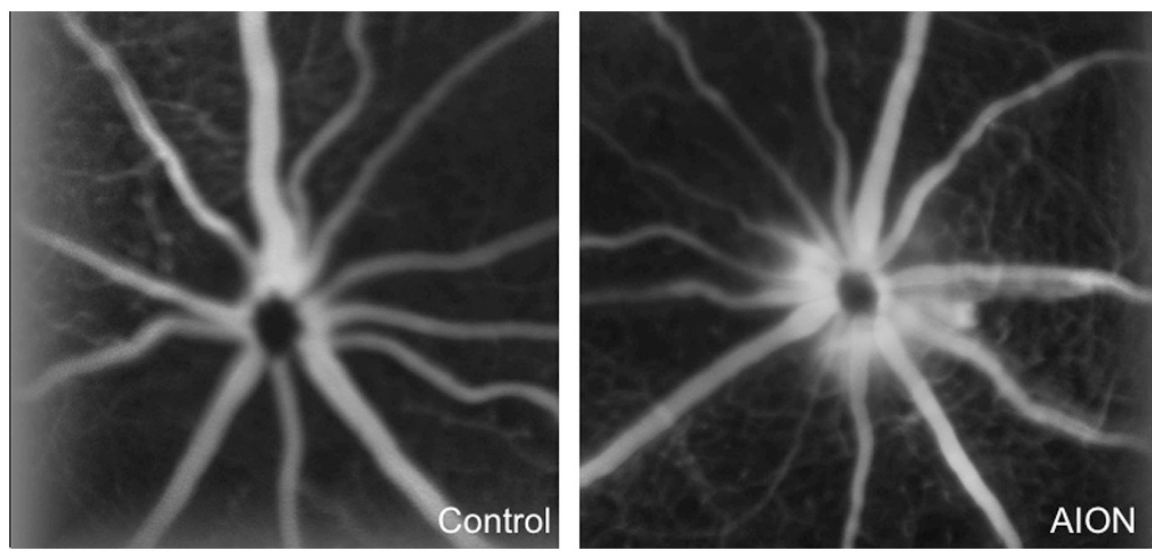

Figure 3 Representative fluorescein angiography performed $30 \mathrm{~min}$ following experimental AION in an adult mouse revealed late staining of the optic disc (right) similar to that of human AION.
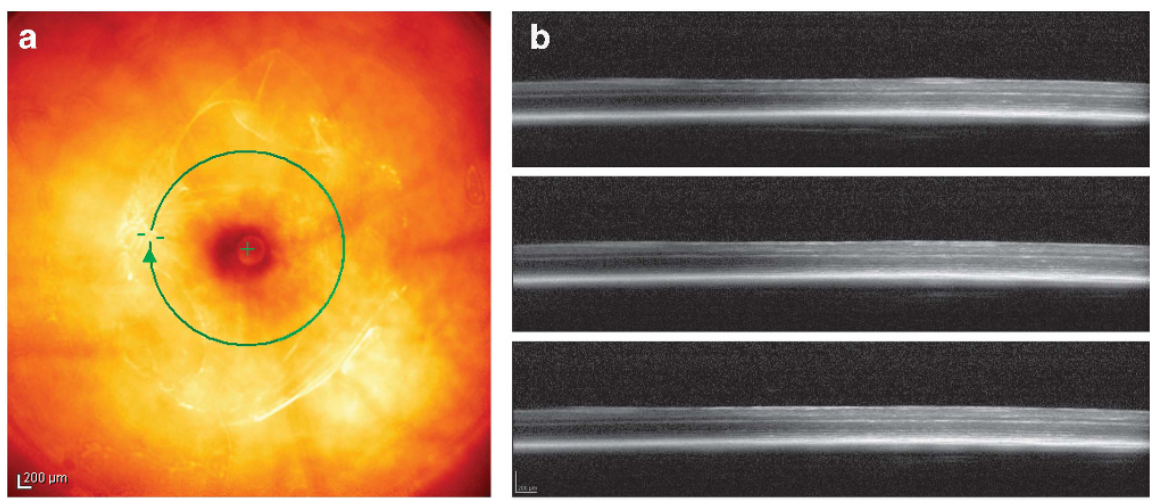

Figure 4 Circular scan did not reveal evidence of subretinal fluid. (a) Fundus photograph with superimposed circular scan line. (b) Representative example of the B-scan.

fluid ( $N=14$, Figure 4). In contrast, the 25-line or posterior pole volume scans through the optic disc revealed subretinal fluid and mild intra-retinal edema in all eyes (Figure 5).

Using a qualitative analysis, we found that $14 \%$ of eyes showed a small amount of fluid, $36 \%$ a moderate amount, and 50\% a large amount of fluid. The fluid accumulated superiorly in $50 \%$, inferiorly in $42 \%$, or both in $7 \%$. The fluid was generally not located immediately adjacent to the disc, which was likely why it was missed in the circular scans. No subretinal fluid was seen at baseline or at 1 week after AION. Quantitative analysis found an average of $323208 \pm 77697 \mu \mathrm{m}^{3}$ of fluid in AION eyes, and no fluid was observed in the control eyes on day $1(P<0.0001)$ (Figure $5 b)$.

\section{Discussion}

We report here an illustrative clinical case of NAION with prominent optic nerve head swelling, intra-retinal edema, and sub-foveolar fluid accumulation. To further explore this phenomenon, we performed SD-OCT analyses of murine experimental AION and found that intra-retinal swelling and subretinal fluid were common in murine AION. The subretinal fluid was prominent 1 day after ischemia and resolves by week 1 . The SD-OCT volume scan was significantly superior to the circular scan in the detection of the subretinal fluid because the fluid did not accumulate immediately adjacent to the optic disc and the volume scan covered a larger area in the posterior pole.

The finding of subretinal fluid in humans has been previously described in one study of patients. ${ }^{6}$ In this series of 76 patients with NAION, about half had some amount of subretinal fluid, and $10 \%$ had subfoveal fluid. Our clinical case demonstrates this finding well. The subretinal fluid accumulation is likely multifactorial. The ischemic optic nerve head and, sometimes, the surrounding retina become leaky with the breakdown of the blood retinal barrier, and this swelling and fluid overwhelm the capacity of the retinal pigment epithelium to reabsorb the excess fluid, leading to fluid 

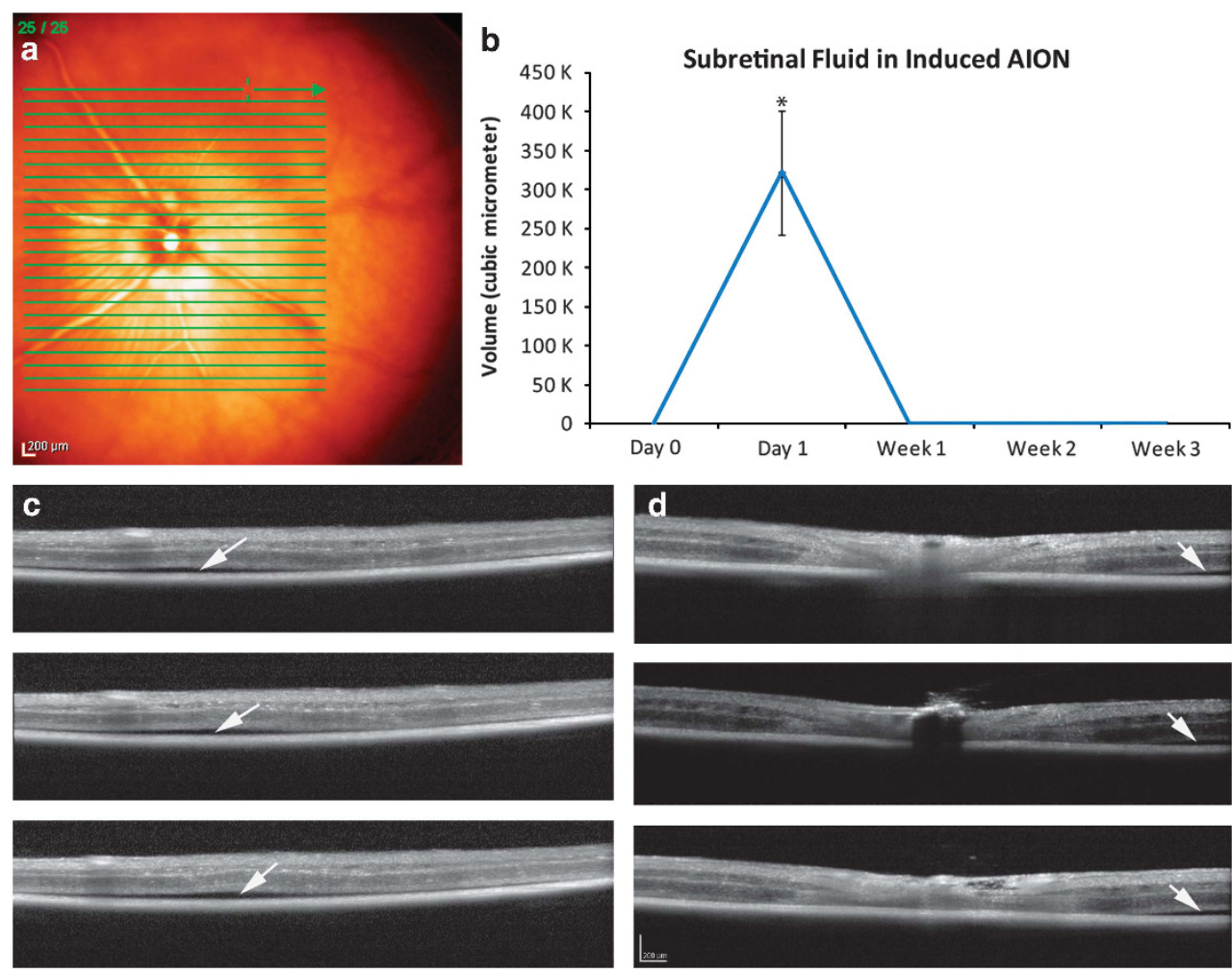

Figure 5 Twenty-line volume scans of the optic disc revealed subretinal fluid in 100\% of eyes. The fluid accumulated superiorly, inferiorly, or both, and typically not immediately adjacent to the disc. (a) Fundus photograph with superimposed 25 B-scan lines. (b) Bar graph showing time course of subretinal fluid collection. (c) Example of moderate amount of subretinal fluid (arrows). These are from the top three green B-scan lines in (a). (d) Example of peripapillary area with small amount of subretinal fluid (arrows) from another mouse.

accumulation in the subretinal potential space. ${ }^{8}$ Muller cells and impaired venous return may also have a role. As the edema decreases over time, the fluid is absorbed and resolves. This could account for some of the improvement in vision that is typical of the clinical course of AION. ${ }^{6}$

Our murine model of photochemical thrombosis seems to reproduce in mice the clinical findings seen after AION in humans with similar findings on histology, SDOCT, and fluorescein angiography. As compared with humans, there seems to be a higher rate of subretinal fluid present in experimental AION, with all the treated animals developing subretinal fluid on day 1 . This greater prevalence in murine AION may be partly related to superior control in the timing of animal studies, as patient OCT studies are typically done at variable times from symptom onset and small amount of fluid could have resolved prior to OCT studies. ${ }^{6}$ The complete reabsorption of fluid by week 1 in murine AION indicates that the murine pigmented epithelium can rapidly balance the vascular leakiness caused by experimental AION. Overall, we have found that murine AION is an excellent model of human
AION and provides a mechanism to characterize important aspects of disease and for studying potential therapies.

Despite its prevalence, there is no indication that the subretinal fluid and retinal edema seen in human and murine AION necessitate clinical intervention. The fluid is likely spillover from optic disc edema and resolves spontaneously without exacerbating the ischemia. Subretinal fluid and retinal ischemia are sometimes treated with anti-VEGF therapies such as in severe cases of diabetic retinopathy or central retinal vein occlusion. Rarely, human AION is treated with anti-VEGF or steroids. ${ }^{17,18}$ Certainly, ischemia could lead to the upregulation of VEGF and perhaps contribute to the formation of subretinal fluid. However, anti-VEGF therapy has been associated with exacerbation of retinal ischemia. ${ }^{19}$ We feel that the benefit of such injections would be outweighed by the risks, especially given that neovascularization is not a common complication of nonarteritic AION.

Although the circular scan mode of the SD-OCT and segmentation of the retinal nerve fiber layer is the most commonly scan used to track patients with optic 
neuropathies in general, the circular scan alone does not adequately detect the peripapillary retinal changes and the presence of subretinal fluid in acute AION. The volume scan significantly increases the sensitivity of detecting retinal changes and subretinal fluid. The reason for this relative poor sensitivity of the circular scan is because the measurement is taken relatively close to the optic nerve head and misses fluid that accumulates further out. On the basis of our clinical and experimental findings, we now routinely perform circular retinal nerve fiber layer scan and macular volume scan to assess patients with AION. We also commonly use the 5-line scan, which shows continuous data from the optic disc through the macula and is particularly helpful to simultaneously assess subretinal and subfoveal fluid.

Recent SD-OCT software advancement has also allowed measurements of the macular ganglion cell complex, which reflects early retinal ganglion cell layer thinning better than the retinal nerve fiber layer analysis in acute and subacute AION due to superimposed optic disc swelling. Although this analysis has been reported to be less affected by optic disc edema compared with the circular scan, the presence of retinal edema and subretinal fluid in some patients with AION means that all OCT scans and automatic calculation of retinal thickness near the optic disc can potentially be affected by edema, and the clinicians need to evaluate the B-scans rather than relying on automatic software calculation of the retinal thickness measurements. ${ }^{20}$ Despite these caveats, we anticipate that the SD-OCT macular scans will be used more routinely in cases of AION, and we speculate that a larger than previously described portion of patients may have some amount of retinal edema or subretinal fluid. A large, prospective study correlating visual function with OCT analysis will help to determine if the retinal edema and subretinal fluid are of significant impact to vision in AION and warrant therapeutic intervention.

\section{Summary}

What was known before

- Some patients with anterior ischemic optic neuropathy develop subretinal fluid.

- Murine models of anterior ischemic optic neuropathy exist.

What this study adds

- A detailed clinical case of subretinal fluid after anterior ischemic optic neuropathy.

- The finding of subretinal fluid in $100 \%$ of mice that underwent experimental anterior ischemic optic neuropathy.

- Volume scan is superior to circular scan in the detection of subretinal fluid in murine anterior ischemic optic neuropathy.

\section{Conflict of interest}

The authors declare no conflict of interest.

\section{Acknowledgements}

YJL was supported by the Career Award in Biomedical Sciences from the Burroughs Wellcome Foundation, Weston Havens Foundation Grant, and Stanford Center for Biomedical Imaging Grant. JKH was supported by the Stanford Medical Scholars Program.

\section{References}

1 Arnold AC. Pathogenesis of nonarteritic anterior ischemic optic neuropathy. J Neuroophthalmol 2003; 23: 157-163.

2 Hayreh SS. Ischemic optic neuropathy. Prog Retin Eye Res 2009; 28: 34-62.

3 Potarazu SV. Ischemic optic neuropathy: models for mechanism of disease. Clin Neurosci 1997; 4: 264-269.

4 Arnold AC, Hepler RS. Natural history of nonarteritic anterior ischemic optic neuropathy. J Neuroophthalmol 1994; 14: 66-69.

5 Yee RD, Selky AK, Purvin VA. Outcomes of optic nerve sheath decompression for nonarteritic ischemic optic neuropathy. J Neuroophthalmol 1994; 14: 70-76.

6 Hedges 3rd TR, Vuong LN, Gonzalez-Garcia AO, Mendoza-Santiesteban CE, Amaro-Quierza ML. Subretinal fluid from anterior ischemic optic neuropathy demonstrated by optical coherence tomography. Arch Ophthalmol 2008; 126: 812-815.

7 Demirel S, Batioglu F, Oguz YG, Ozmert E. Macular involvement and response to steroid therapy demonstrated by optical coherence tomography in non-arteritic anterior ischaemic optic neuropathy. Neuro-Ophthalmology 2013; 37: 161-164.

8 Marmor MF. Mechanisms of fluid accumulation in retinal edema. Doc Ophthalmol 1999; 97: 239-249.

9 Bernstein SL, Johnson MA, Miller NR. Nonarteritic anterior ischemic optic neuropathy (NAION) and its experimental models. Prog Retin Eye Res 2011; 30: 167-187.

10 Bernstein SL, Guo Y, Kelman SE, Flower RW, Johnson MA. Functional and cellular responses in a novel rodent model of anterior ischemic optic neuropathy. Invest Ophthalmol Vis Sci 2003; 44: 4153-4162.

11 Chen CS, Johnson MA, Flower RA, Slater BJ, Miller NR, Bernstein SL. A primate model of nonarteritic anterior ischemic optic neuropathy. Invest Ophthalmol Vis Sci 2008; 49: 2985-2992.

12 Slater BJ, Mehrabian Z, Guo Y, Hunter A, Bernstein SL. Rodent anterior ischemic optic neuropathy (rAION) induces regional retinal ganglion cell apoptosis with a unique temporal pattern. Invest Ophthalmol Vis Sci 2008; 49: 3671-3676.

13 Goldenberg-Cohen N, Guo Y, Margolis F, Cohen Y, Miller NR, Bernstein SL. Oligodendrocyte dysfunction after induction of experimental anterior optic nerve ischemia. Invest Ophthalmol Vis Sci 2005; 46: 2716-2725.

14 Pangratz-Fuehrer S, Kaur K, Ousman SS, Steinman L, Liao YJ. Functional rescue of experimental ischemic optic neuropathy with alphaB-crystallin. Eye (Lond) 2011; 25: 809-817. 
15 Ho JK, Stanford M, Shariati MA, Dalal R, Liao YJ. Optical coherence tomography study of experimental anterior ischemic optic neuropathy and histologic confirmation. Invest Ophthalmol Vis Sci 2013; 54(9): 5981-5988.

16 Sagdullaev BT, DeMarco PJ, McCall MA. Improved contact lens electrode for corneal ERG recordings in mice. Doc Ophthalmol 2004; 108: 181-184.

17 Atkins EJ, Bruce BB, Newman NJ, Biousse V. Treatment of nonarteritic anterior ischemic optic neuropathy. Surv Ophthalmol 2010; 55: 47-63.
18 Hayreh SS. Non-arteritic anterior ischemic optic neuropathy: role of systemic corticosteroid therapy. Suro Ophthalmol 2010; 55: 399-400.

19 Manousaridis K, Talks J. Macular ischaemia: a contraindication for anti-VEGF treatment in retinal vascular disease? Br I Ophthalmol 2012; 96: 179-184

20 Kardon RH. Role of the macular optical coherence tomography scan in neuro-ophthalmology.

J Neuroophthalmol 2011; 31: 353-361. 